\title{
Inactivation of ribosomal protein S27-like confers radiosensitivity via the Mdm2-p53 and Mdm2-MRN-ATM axes
}

\author{
Yongchao Zhao ${ }^{1,2,3}$, Mingjia Tan' ${ }^{2}$ Xia Liu' ${ }^{1}$ Xiufang Xiong ${ }^{1,2}$ and Yi Sun ${ }^{1,2,4}$
}

\begin{abstract}
RPS27L (ribosomal protein S27-like) is an evolutionarily conserved ribosomal protein and a direct p53 target. We recently reported that Rps 271 disruption triggers ribosomal stress to induce p53, causing postnatal death, which can be

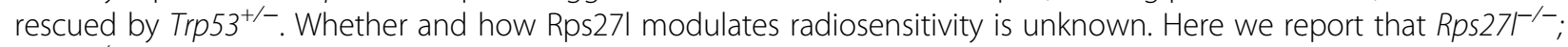
$\operatorname{Trp}_{53^{+/-}}$mice are extremely sensitive to radiation due to reduced proliferation and massive induction of apoptosis in radiation-sensitive organs. Mechanistically, the radiation sensitivity is mediated by two signaling pathways: (1) activated p53 pathway due to imbalanced Mdm2/Mdm4 levels and reduced E3 ligase activity; and (2) reduced DNA damage response due to reduced MRN/Atm signal as a result of elevated Mdm2 binding of Nbs1 to inhibit Nbs1-Atm binding and subsequent Atm activation. Indeed, heterozygous deletion of Mdm2 restores the MRN/Atm signal. Collectively, our study revealed a physiological condition under which Rps27l regulates the Mdm2/p53 and MRN/Atm axes to maintain DNA damage response and to confer radioprotection in vivo.
\end{abstract}

\section{Introduction}

The genome of a cell is constantly damaged by internal metabolites and environmental insults, such as ultraviolet light, ionizing radiation (IR), drug exposure, and oxidative stress $^{1}$. To ensure maintenance of genomic stability, cells have evolved DNA damage response (DDR), a global signaling network to sense DNA damage and trigger distinct cellular responses including DNA repair, cell cycle arrest, senescence, and apoptosis ${ }^{2,3}$.

Specifically, upon DNA damage with DSBs (doublestranded breaks), Mre11-Rad50-Nbs1 (MRN) complex acts as a sensor to recruit ATM to the damage sites by directly binding of ATM with $\mathrm{C}$ terminus of $\mathrm{Nbs}^{4-6}$. ATM is subsequently activated via autophosphorylation,

\footnotetext{
Correspondence: Yongchao Zhao (yongchao@zju.edu.cn) or

Xiufang Xiong (xiufang@zju.edu.cn) or Yi Sun (yisun@zju.edu.cn)

${ }^{1}$ Institute of Translational Medicine, Zhejiang University School of Medicine, Hangzhou, China

2Division of Radiation and Cancer Biology, Department of Radiation Oncology, University of Michigan, Ann Arbor, MI, USA

Full list of author information is available at the end of the article Edited by M. Malewicz
}

\section{(c) The Author(s). 2018}

which in turn phosphorylates its downstream effectors, including all three members of MRN complex, Chk2, H2AX, p53, and BRCA1 among others, to mediate distinct downstream responses ${ }^{7}$. In contrast, ATR is activated by damage-induced Replication Protein A (RPA)coated single-stranded DNA and DNA replication stress to directly phosphorylate and activate Chk1 and other substrates. As a result, activated ATM-Chk2 and ATR-Chk1 pathways maintain genomic stability by triggering multiple cellular responses ${ }^{8,9}$.

Ribosomal proteins (RPs) are not only the essential components of the ribosome, but also play important roles in assembly of ribosome particles, a process termed as ribosome biogenesis ${ }^{10}$. Perturbation of ribosome biogenesis by multiple stresses, such as DNA damage, RP mutations, drug insults, nutrient deprivation, or oncogenic activation triggers nucleolar stress, also known as ribosomal stress (for review, see ref. 11). In response to ribosomal stress, a number of RPs were found to release from ribosome and directly bind with MDM2 to inhibit its ligase activity towards p53, leading to p53 activation,

\footnotetext{
Open Access This article is licensed under a Creative Commons Attribution 4.0 International License, which permits use, sharing, adaptation, distribution and reproduction changes were made. The images or other third party material in this article are included in the article's Creative Commons license, unless indicated otherwise in a credit line to the material. If material is not included in the article's Creative Commons license and your intended use is not permitted by statutory regulation or exceeds the permitted use, you will need to obtain
} permission directly from the copyright holder. To view a copy of this license, visit http://creativecommons.org/licenses/by/4.0/. 
followed by p53-dependent cell cycle arrest and apoptosis (for reviews, see refs. 12-15). Given p53 acting as a guardian of the genome ${ }^{16}$, RPs could play significant roles in maintenance of genome integrity in a p53-dependent manner.

RPS27L (NM_015920) is an evolutionarily conserved ribosomal protein with 84-amino acid, which differs from its family member RPS27 (NM_001030) only by three amino acids (R5K, L12P, K17R). We and the others have previously reported that RPS27L is a direct p53 transcriptional target ${ }^{17,18}$. Our recent in vivo study showed that Rps27l disruption triggers ribosomal stress to stabilize Mdm2, which degrades Mdm4 to reduce Mdm2Mdm4 E3 ligase activity towards p53, leading to p53dependent apoptotic depletion of hematopoietic stem cells and postnatal death, which can be rescued by heterozygous deletion of $\operatorname{Trp} 53^{19}$. Rps $27 l$ deletion also enhances lymphomagenesis in $\operatorname{Tr} p 53^{+/-}$background by causing genomic instability to selectively lose Trp53 heterozygosity $^{19}$. However, whether and how Rps27l affects the efficiency of DNA damage response and modulates radiosensitivity in vivo is previously unknown.

Here we report that inactivation of Rps27l in a Trp $53^{+/-}$ background significantly enhances the sensitivity of mice to ionizing radiation as a consequence of reduced proliferation and massive apoptosis in multiple radiation-sensitive organs. Involving mechanisms include (1) imbalanced levels of Mdm2 and Mdm4, leading to subsequent p53 activation and (2) elevated Mdm2 binding of Nbs1 to abrogate MRN complex for ATM activation, leading to reduced DNA damage response. Thus, Rps27l regulates radiation sensitivity in both p53-dependent and p53independent manners and might serve as an attractive target for radiosensitization.

\section{Results}

Rps27l inactivation sensitizes $\operatorname{Trp} 53^{+/-}$mice to radiation

Our previous study showed that Rps27l regulates genomic stability ${ }^{19}$, whose disruption causes postnatal death, which can be rescued by heterozygous deletion of Trp53. The viability of $R p s 27 l^{-/-}$; $\operatorname{Trp} 53^{+/-}$mice provided us an opportunity to study the role of Rps27l in radiation-induced tumorigenesis. We treated three genotypes of $R p 27 l$ mice, all under the $\operatorname{Tr} p 53^{+/-}$background with whole-body radiation at the dose of 4 Gy. Unexpectedly, we found that $R p s 277^{-/-}$; $\operatorname{Tr} p 53^{+/-}$mice are very sensitive to radiation with a median survival of 108 days and all death at 150 days before tumor development. The difference is statistically significant $(p<0.0001)$, as compared to $R p s 27 \mathrm{l}^{+/+}$; $\operatorname{Tr} p 53^{+/-}$or $R p s 2 l^{+/-}$; Trp $53^{+/-}$mice, which have no statistical difference between each other $(p>0.05)$ (Fig. 1a). High radiosensitivity was further confirmed when $R p s 27 l^{-1-}$; $\operatorname{Trp} 53^{+/-}$mice was exposed to $8 \mathrm{~Gy}$ radiation, which led to a median survival of 14.5 days and all death at 28 days. Again, the difference is statistically significant from $R p s 27 l^{+/+}$; $\operatorname{Trp} 53^{+/-}$or $R p s 27 l^{+/-}$; $\operatorname{Tr} p 53^{+/-}$mice $(p=$ 0.0076) (Fig. 1b).

We next determined the cause(s) of mouse death by 8 Gy radiation with focus on cell proliferation by BrdU
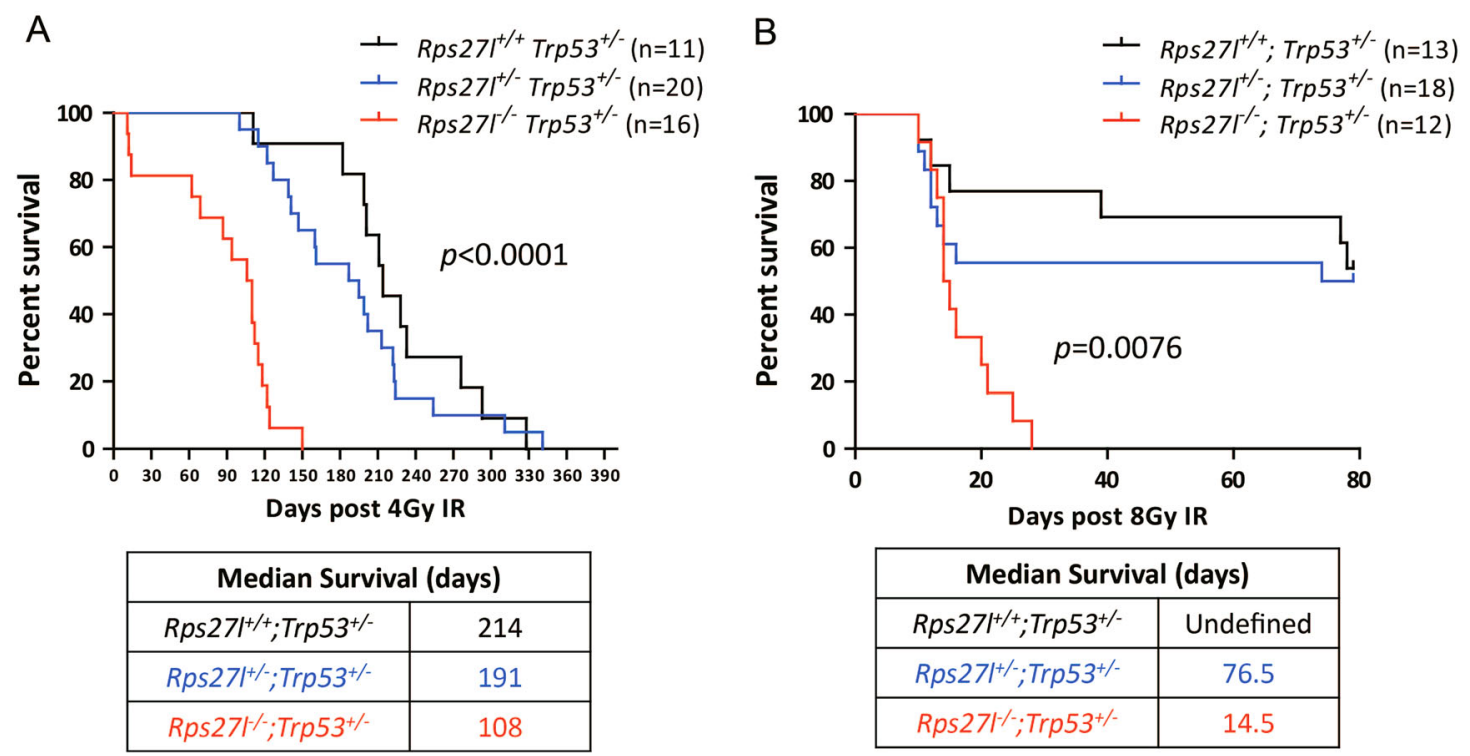

Fig. 1 Rps27l inactivation sensitizes $\operatorname{Trp53^{+/-}}$ mice to radiation. Inactivation of Rps27l shortens the life span of $\operatorname{Trp} 53^{+/-}$mice after whole-body radiation. Three groups of mice at age of 5 weeks $\mathbf{a}$ or $8-10$ weeks $\mathbf{b}$ with indicated genotypes were irradiated at 4 Gy (a) and 8 Gy (b), respectively, followed by survival monitor. Kaplan-Meier survival curves were shown. Log-rank test, statistical $p$ values were shown 

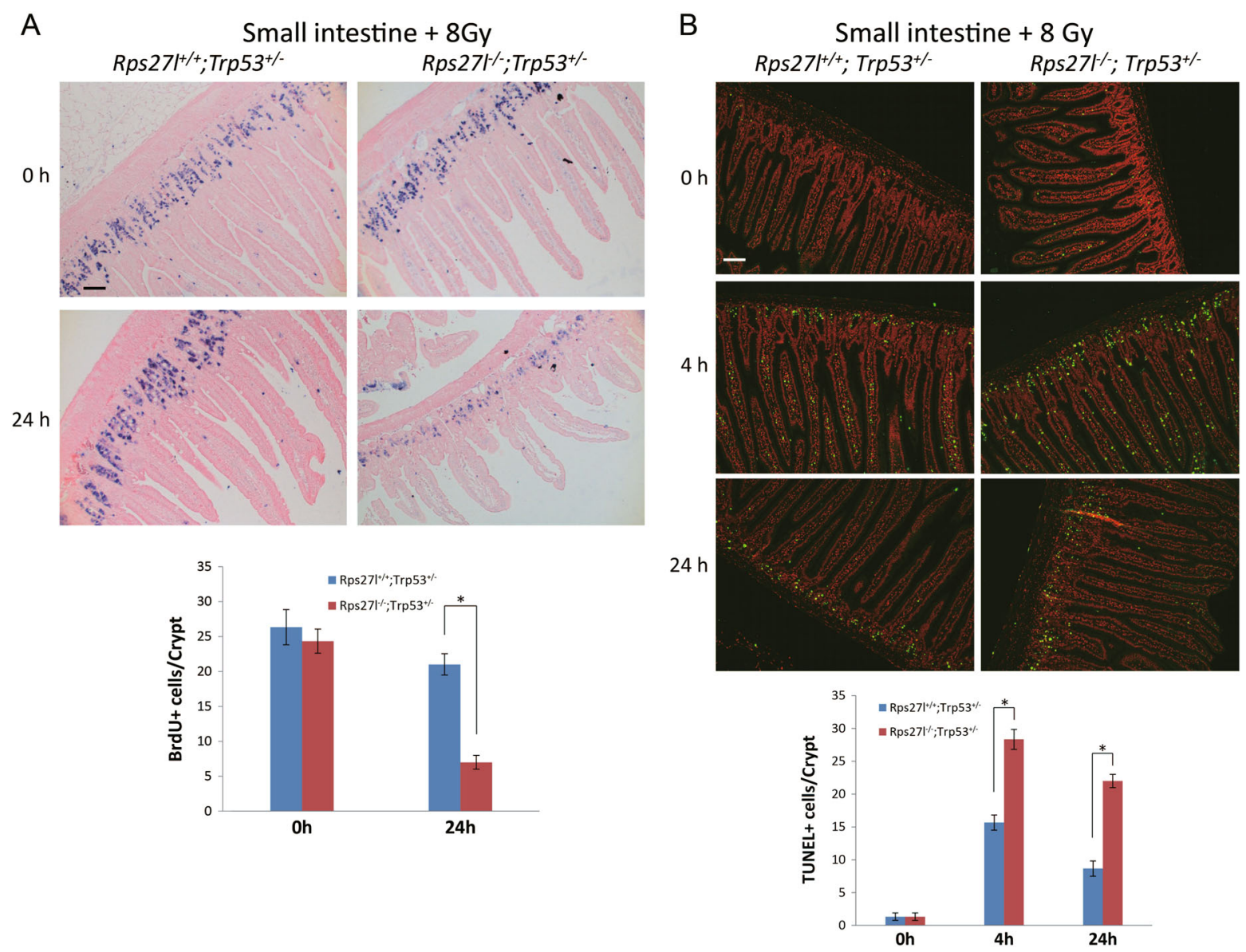

Fig. 2 Rps 27 inactivation reduces proliferation and enhances apoptosis upon radiation. Inactivation of Rps27/ inhibits proliferation and induces apoptosis upon radiation in small intestine. BrdU was i.p. injected into WT littermates and Rps $27 \mathrm{l}$ mutants $2 \mathrm{~h}$ before mice being sacrificed. Small intestine tissues were harvested, followed by BrdU staining $\mathbf{a}$ and TUNEL assay $\mathbf{b}$. Positive staining cells of each crypt were counted from at least three randomly selected microscopic fields from three independent mice (lower panels). ${ }^{*} p<0.05$. Scale bars represent $100 \mu \mathrm{m}$

incorporation and apoptosis by TUNEL and cleaved caspase-3 staining assays in radiation-sensitive organs, including small intestine, thymus and spleen. Compared to $R p s 27 l^{+/+}$; $\operatorname{Trp} 53^{+/-}$mice, $R p s 27 l^{-/-}$; $\operatorname{Tr} p 53^{+/-}$mice showed significantly reduced proliferation in small intestine (Fig. 2a) and spleen (Figure S1A) $24 \mathrm{~h}$ post radiation. Remarkably increased apoptosis was readily observed 4 or $24 \mathrm{~h}$ post $8 \mathrm{~Gy}$ of radiation using TUNEL assay (Fig. 2b), while only a moderate increase of cleaved caspase-3 positively stained cells was seen $4 \mathrm{~h}$ post $15 \mathrm{~Gy}$ of radiation in small intestine (Figure S1B) due to the sensitivity of different assays and tissues. Indeed, we observed more cleaved caspase- 3 positively stained cells 4 or 24 h post 8 Gy of radiation in thymus and spleen (Figure S1C and D). We further employed clonogenic assay to analyze the radiosensitivity of immortalized MEFs, and found that Rps $27 l^{-1-}$; $\operatorname{Trp} 53^{+/-}$MEFs were more sensitive to radiation than $R p s 27 \mathrm{l}^{+/+}$; $\operatorname{Trp} 53^{+/-}$MEFs (Figure S1E). Collectively, $R p s 27 l$ inactivation confers radiosensitivity in
$\operatorname{Trp} 53^{+/-}$mice with reduced proliferation and enhanced apoptosis in radiation-sensitive organs.

Rps27l inactivation increases p53 activity in $\operatorname{Trp} 53^{+/-}$mice To investigate potential molecular mechanisms by which Rps27l inactivation confers radiosensitivity in $\operatorname{Trp}_{53^{+/-}}$mice, we first focused on $\mathrm{p} 53$, since (1) tumor suppressor p53 is a key regulator of cellular response to radiation $^{20}$, and (2) our recent study showed that $R p s 27$ disruption causes a moderate increase of p53 and its targets in multiple organs, including spleen, bone marrow and fetal liver as well as MEFs ${ }^{19}$. Indeed, as compared to $R p s 27 l^{+/+}$; $\operatorname{Trp} 53^{+/-}$littermates, $R p s 27 l^{-/-}$; $\operatorname{Trp} 53^{+/-}$ mice showed higher p53 levels under unstressed condition in small intestine (Fig. 3a, b), spleen (Fig. 3c), thymus (Figure S2A), and bone marrow (Figure S2B) by immunostaining. Higher p53 levels were also found in spleen, thymus, and testis from two pairs of $\mathrm{Rps}_{27 \mathrm{I}^{-/-}}$; $\operatorname{Trp} 53^{+/-}$ mice by immunoblotting (Fig. 3d). Upon ionizing 


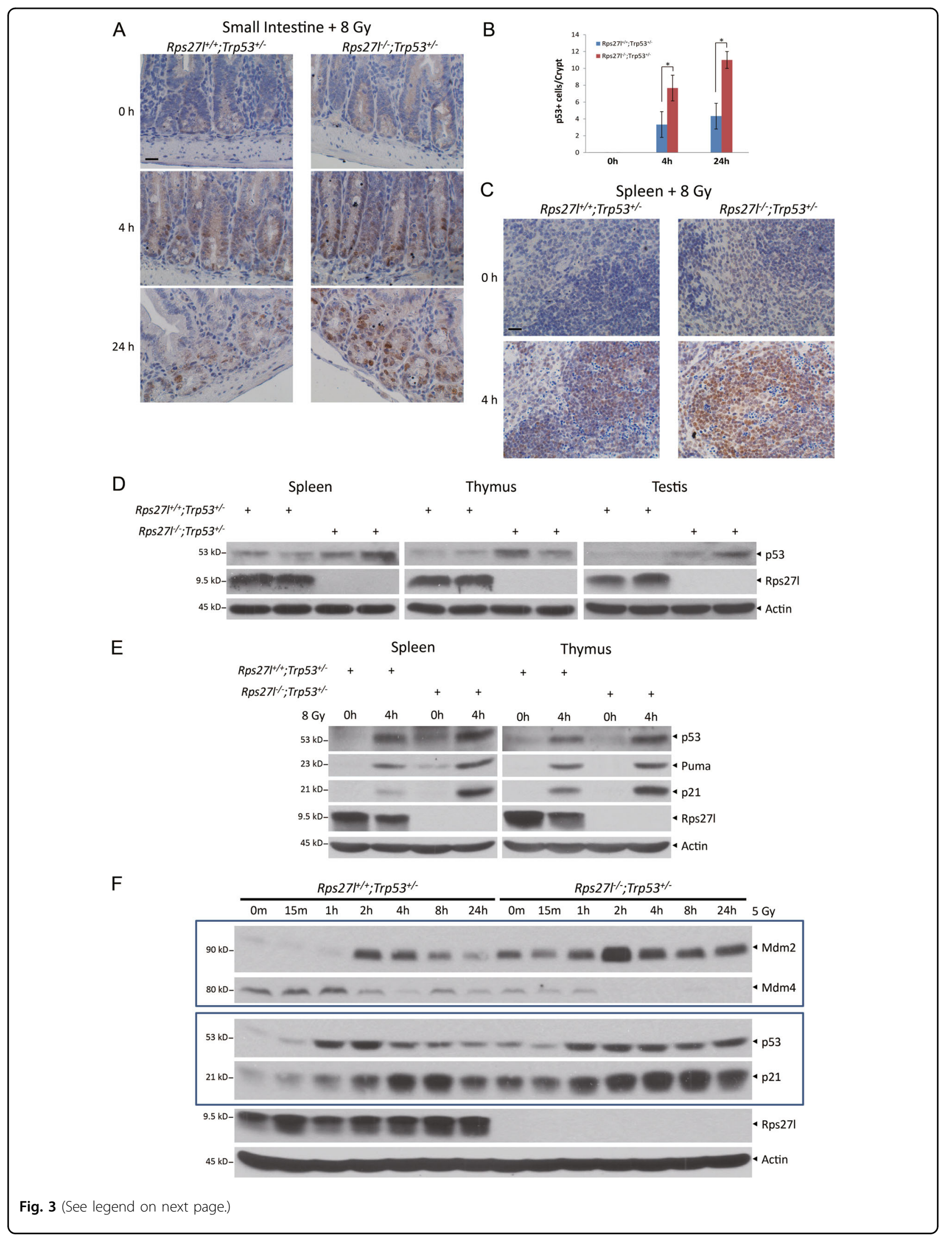


(see figure on previous page)

Fig. 3 Rps27l inactivation increases the levels of p53 and its targets in Trp53 ${ }^{+/-}$mice. a-c Inactivation of Rps27l induces the levels of p53 in small intestine $(\mathbf{a}, \mathbf{b})$ and spleen (c) of $\operatorname{Trp} 53^{+/-}$mice upon radiation. Mice with indicated genotypes were irradiated at $8 \mathrm{~Gy}$, and then small intestine $(\mathbf{a}, \mathbf{b})$ and spleen (c) were collected at indicated time points, followed by IHC staining with p53 antibody and photograph. Positive staining cells of each crypt were counted from at least three randomly selected microscopic fields (b). ${ }^{*} p<0.05$. Scale bars represent $20 \mu \mathrm{m}$. $\mathbf{d}$, e Inactivation of Rps 271 induces $\mathrm{p} 53$ levels in multiple organs of Trp53 $3^{+/}$mice with or without radiation. The indicated organs from two individual male mice with indicated genotypes were homogenized and subjected to IB with indicated antibodies. $\mathbf{f}$ Inactivation of Rps 27 induces the levels of p53 and its targets in $\operatorname{Trp} 53^{+/-}$MEFs upon radiation. MEFs with indicated genotypes were irradiated at $5 \mathrm{~Gy}$. Cells were then harvested at indicated time points post irradiation, followed by IB with indicated antibodies

radiation, increased levels of p53 and its two well-known targets p 21 and Puma were found in spleen and thymus from $R p s 277^{-/-}$; $\operatorname{Tr} p 53^{+/-}$mice, as compared to those from $R p s 27 l^{+/+}$; Trp53 $3^{+/-}$mice (Fig. 3e).

Our previous study revealed that under physiological $\operatorname{Trp} 3^{+/+}$background, Rps 27 inactivation triggers ribosomal stress to stabilize Mdm2, which degrades Mdm4 to reduce Mdm2-Mdm4 E3 ligase towards p53, leading to p53 accumulation and activation ${ }^{19}$. We extended this observation to $\operatorname{Trp} 53^{+/-}$MEFs and found that compared to wild-type cells, the levels of Mdm2 or Mdm4 in Rps27lnull cells were increased or decreased, respectively, upon radiation with consequent increased duration of p53 stabilization and p21 levels (Fig. 3f). Thus, Rps27l deletion under $\operatorname{Trp} 53^{+/-}$background alters the levels of Mdm2 and Mdm4, leading to activation of p53 and its downstream targets in multiple radiation-sensitive organs, followed by reduced proliferation and enhanced apoptosis, and eventually radiosensitization.

\section{Rps27I inactivation impairs DNA damage response to radiation}

We next determined if radiosensitization by Rps27l inactivation under $\operatorname{Tr} 553^{+/-}$background can also be attributable to altered DNA damage response to radiation. We first compared activation of Atm and Chk1 between paired $R p s 27 l^{+/+}$; $\operatorname{Trp} 53^{+/-}$and $\operatorname{Rps} 277^{-/-}$; $\operatorname{Trp} 53^{+/-}$ MEFs at multiple time points post radiation, and found reduced activation, as reflected by reduced levels of Atm and Chk1 phosphorylation in Rps27l ${ }^{-/-}$MEFs (Fig. 4a). Consistently, by immunofluorescent staining and immunoblotting, we detected the lower levels of $\gamma \mathrm{H} 2 \mathrm{AX}$, a hallmark of DNA damage, in Rps $277^{-/-}$; Trp $53^{+/-}$MEFs at multiple time points post radiation (Fig. 4b, c). Moreover, impaired activation of Atm and Chk1 was also seen in


doses of radiation (Fig. 4d), or to different chemotherapeutic agents, such as VM-26 (Fig. 4e) and doxorubincin (Fig. 4f).

We extended $\gamma \mathrm{H} 2 \mathrm{AX}$ immunofluorescent staining to mouse organs and found that the number of $\gamma \mathrm{H} 2 \mathrm{AX}$ positively stained cells was significantly reduced in the crypts of small intestine (Fig. 5a) and bone marrow
(Fig. 5b) of $R p s 27 \mathrm{I}^{-/-}$; $\operatorname{Trp} 53^{+/-}$mice upon radiation exposure. Thus, Rps27l inactivation impairs Atm activation and subsequent DNA damage response to genotoxic agents.

\section{Rps27l inactivation impairs Atm activation via increased Mdm2 binding of Nbs1}

MDM2 was reported to negatively affect genomic stability independent of p53 by directly binding to Nbs1 of the Mre11-Rad50-Nbs1 (MRN) complex, thus impairing ATM activation and subsequent DNA damage response $^{21-23}$. We found that compared to $\operatorname{Rps}^{2} 7 \mathrm{I}^{+/+}$; Trp $53^{+/-}$MEFs, Rps $27 l^{-/-}$; Trp $53^{+/-}$MEFs had significantly higher Mdm2 levels under unstressed condition or at multiple time points post radiation (Fig. 3f and Fig. 6a). We then determined whether elevated Mdm2 binds more Nbs1 to abrogate MRN-induced Atm activation. Indeed, in two pull-down assays, we detected more Nbs1 in Mdm2 immunoprecipitates and lesser Atm in Nbs1 immunoprecipitates in Rps $27 l^{-/-}$; Trp $53^{+/-}$MEFs than in $R p s 27 l^{+/+}$; Trp $53^{+/-}$MEFs at multiple time points post radiation (Fig. 6b), suggesting increased Mdm2-Nbs1 binding and decreased Nbs1-Atm binding upon Rps27l inactivation. A causal role of $\mathrm{Mdm} 2$ in the process was demonstrated, since simultaneous deletion of one allele of $M d m 2$ restored the impaired DNA damage response by Rps27l inactivation, as reflected by the recovery of the phosphorylated levels of Atm in $R p s 27 l^{-/-}$; Trp $53^{+/-}$; $M d m 2^{+/-}$MEFs (Fig. 6c). Interestingly, phosphorylated Chk1 levels were not significantly higher in $R p s 27 l^{-/-}$; $\operatorname{Trp} 53^{+/-} ; \mathrm{Mdm}^{+/-}$MEFs than in $R p s 27 \mathrm{I}^{-/-}$; $\operatorname{Trp} 53^{+/-}$; $M d m 2^{+/+}$MEFs, suggesting that Mdm2 deletion does not rescue defective ATR activation (Fig. 6c).

Finally, we attempted to determine whether increased $\mathrm{Mdm} 2$ plays a causal role in conferring radiosensitization in vivo by generating mice with background of $R p s 27 l^{-1-}$; $\operatorname{Trp} 53^{+/-} ; M d m 2^{+/-}$. However, heterozygous deletion of Mdm2 increased the basal level of p53 (Fig. 6c), and these mice were short-lived with $80 \%$ of them died within 6 weeks after birth (Figure S3), further suggesting a feedback loop of the Mdm2/Mdm4-p53 axis to precisely regulate the p53 levels. Taken together, our results demonstrate that Rps27l inactivation causes increased 

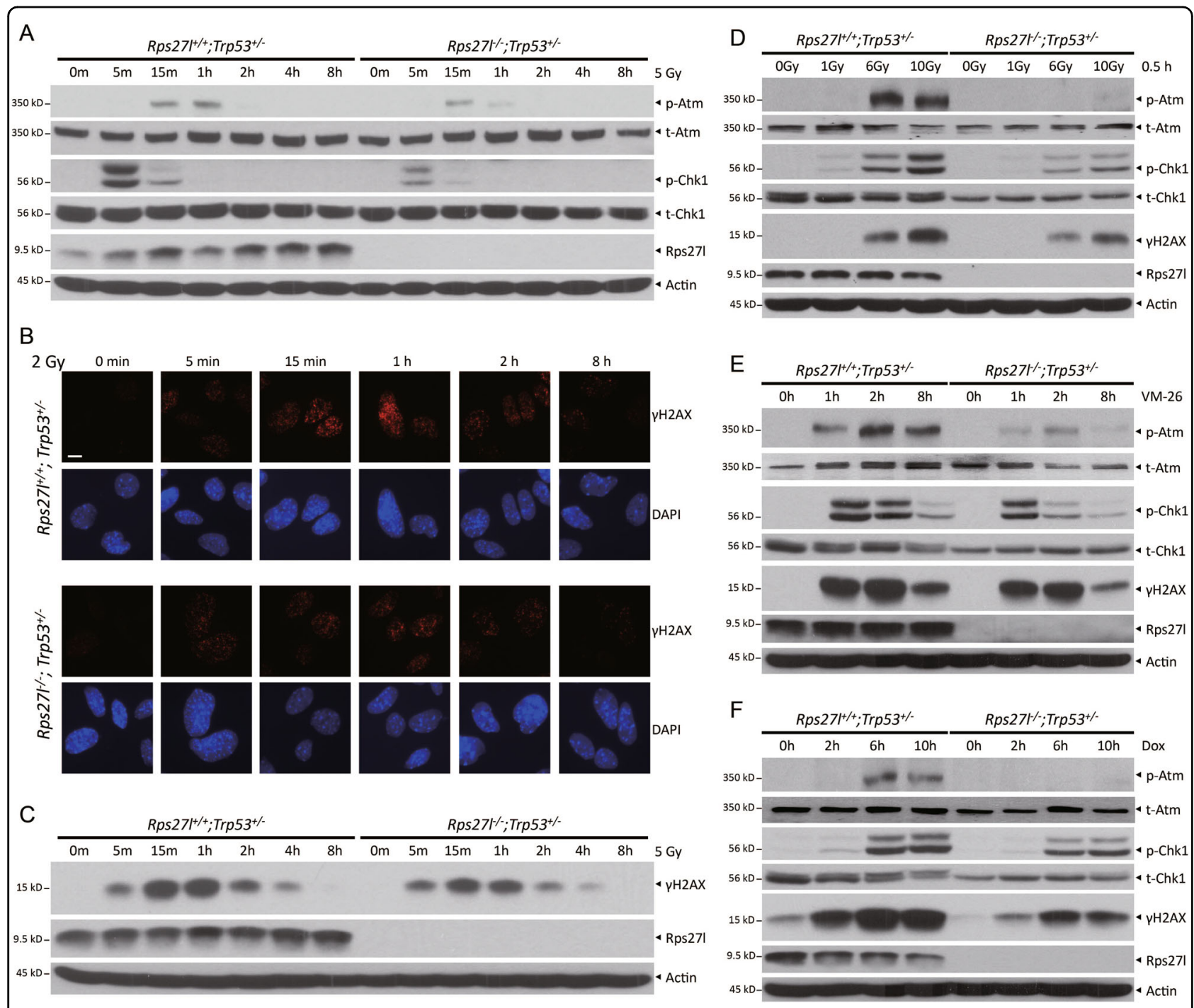

Fig. 4 Rps27l inactivation impairs the activation of Atm and DNA damage response. a-c Inactivation of Rps27/ impairs DNA damage response in $\mathrm{Trp}_{5} 3^{+/-}$MEFs upon radiation. MEFs with indicated genotypes were irradiated for indicated periods of time, followed by IB with indicated antibodies (a, c) or immunofluorescent staining (b). $\mathbf{d}-\mathbf{f}$ Inactivation of Rps 27 I impairs DNA damage response in $\operatorname{Trp5} 3^{+/-}$MEFs upon different doses of radiation (d) or upon treatment with DNA damage agents: $5 \mu \mathrm{M}$ VM-26 (e) or $1 \mu \mathrm{M}$ doxorubincin (Dox) (f) for indicated time periods, followed by IB with indicated antibodies

Mdm2-Nbs1 binding with subsequent decreased Nbs1-Atm binding to block radiation-induced Atm activation, leading to impaired DNA damage response and enhanced sensitivity to radiation.

\section{Discussion}

Several ribosomal proteins, including RPL37 ${ }^{24}, \mathrm{RPS}^{25}$, RPL $26^{26}$, and RPS26 ${ }^{27}$, were reported to play a role in the maintenance of genomic stability through multiple p53dependent mechanisms by stabilizing p53 via $\mathrm{Mdm} 2$ inhibition, directly increasing p53 translation, or promoting p53 transactivation of its downstream targets in response to DNA damage insults ${ }^{15,28}$. Our recent study using a knockout mouse model demonstrated that Rps27l is required for genomic stability under a $\operatorname{Tr} p 53^{+/-}$background and $R p s 27 l^{-/-}$; $\operatorname{Trp} 53^{+/-}$mice eventually develop spontaneous lymphoma after selective deletion of remaining wild-type $\operatorname{Trp} 53$ allele $^{19}$. In this study, we attempted to examine potential role of Rps27l in radiation-induced tumorigenesis and unexpectedly found that inactivation of $R p s 27 l$ greatly sensitizes $\operatorname{Trp} 53^{+/-}$mice to ionizing radiation at both low and high doses (Fig. 1). A careful examination of radiosensitive organs such as spleen, thymus and small intestine of Rps $27 l^{-/-}$; $\operatorname{Trp} 53^{+/-}$mice revealed reduced proliferation and greatly enhanced apoptosis (Fig. 2), indicating that 


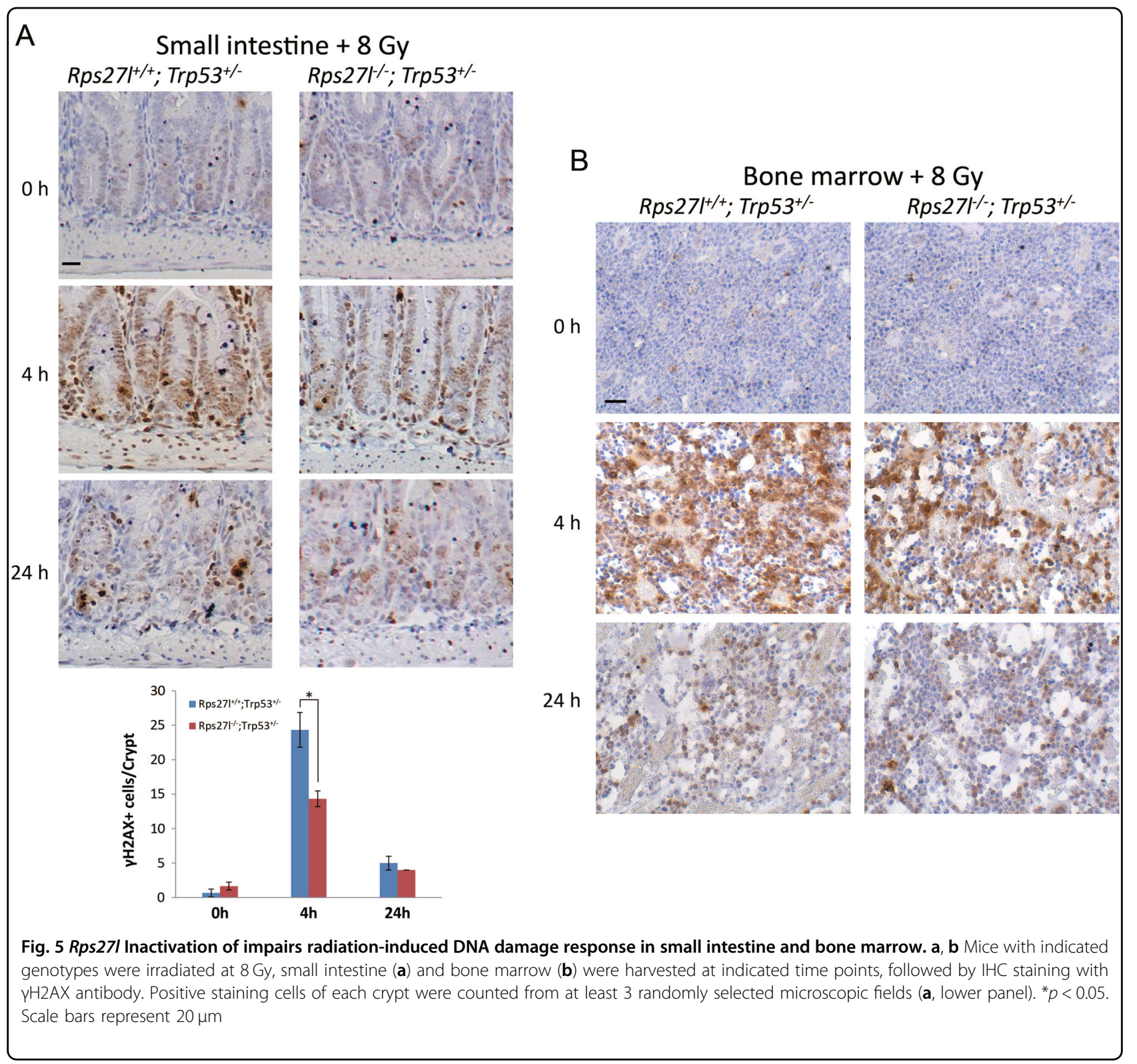

enhanced radiosensitivity is likely due to the failure of these organs.

As a master regulator of cellular responses to stress and a guardian of genome, p53 is precisely regulated to a low level mainly by Mdm2, a major E3 ubiquitin ligase for targeted p53 ubiquitylation and degradation ${ }^{29-31}$, and Mdm4, an Mdm2 family member, which forms a heterodimer with $\mathrm{Mdm} 2$ to have an optimal E3 ligase activity toward $\mathrm{p} 53^{32-34}$. Our previous study showed that the levels of p53 are moderately induced due to reduced Mdm2/Mdm4 E3 ligase activity toward p53 upon Rps27l inactivation, leading to depletion of hematopoietic stem cells via apoptosis and eventual postnatal death ${ }^{19}$. This death phenotype is fully rescued by simultaneous deletion of one allele of $\operatorname{Trp} 53^{19}$. Here we showed that the p53 levels are slightly higher in few organs and MEFs derived from $R p s 27 l^{-/}$; $\operatorname{Trp} 53^{+/-}$mice than those from $R p s 27 l^{+/+}$; $\operatorname{Trp} 53^{+/-}$mice, and can be further induced by DNA damaging agents (Fig. 3) with mechanism again involving Mdm2 accumulation and Mdm4 degradation (Figs. 3, 4 and 6a). Therefore, Rps27l is required to keep p53 levels precisely in check when wild-type p53 is present, regardless with one or both alleles of $\operatorname{Trp} 53$ within a cell. It is worth noting that a slight increase of p53 levels is largely dispensable to the survival of $R p s 27 l^{-/-}$; $\operatorname{Tr} p 53^{+/-}$ mice under unstressed condition, as evidenced by their significant extension of life $\operatorname{span}^{19}$. However, further induction of $\mathrm{p} 53$ levels by radiation exposure (even with a 


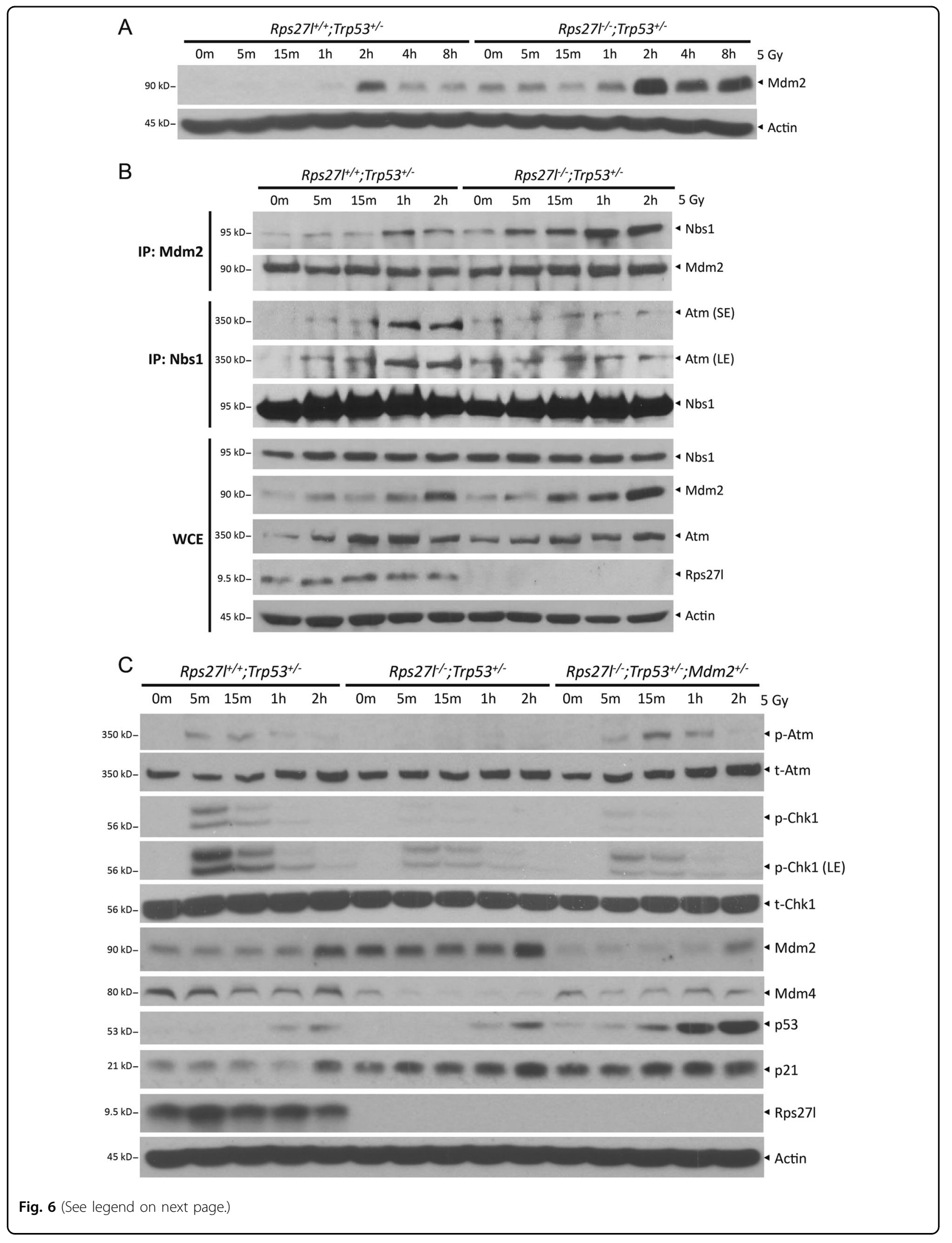


(see figure on previous page)

Fig. 6 Rps27l inactivation impairs the activation of Atm via increased binding of Mdm2 with Nbs1. a Inactivation of Rps $27 /$ induces Mdm2 levels in Trp53 $3^{+-}$MEFs. MEFs with indicated genotypes were left untreated or irradiated at $5 \mathrm{~Gy}$. Cells were then harvested at indicated time points post irradiation, followed by IB with indicated antibodies. $\mathbf{b}$ Inactivation of Rps 27 I increases the binding of Mdm2 with Nbs1 and decreases the binding of Nbs1 with Atm in Trp53 $3^{+/-}$MEFs. MEFs with indicated genotypes were left untreated or irradiated at 5 Gy. Cells were then harvested at indicated time points post irradiation, and subjected to immunoprecipitation with Mdm2 or Nbs1 antibody, followed by IB with indicated antibodies. c Reduction of Mdm2 rescues the impairment of DNA damage response induced by Rps27l inactivation in Trp53 ${ }^{+/-}$MEFs. MEFs with indicated genotypes were left untreated or irradiated at $5 \mathrm{~Gy}$. Cells were then harvested at indicated time points post irradiation, followed by IB with indicated antibodies. SE shorter exposure, LE longer exposure, WCE whole-cell extract

low dose) significantly accelerates death, making it impossible to evaluate radiation-induced tumorigenesis.

We also made a novel observation that Rps27l depletion under $\operatorname{Tr} 553^{+/-}$background greatly reduces DNA damage response to the damaging agents (Figs. 4 and 5). Mechanistically, we found, by using paired MEFs derived from littermate embryos with genotypes of $R p s 27 l^{+/+}$; $\operatorname{Trp} 53^{+/-}$vs. $R p s 27 l^{-/-}$; $\operatorname{Trp} 53^{+/-}$, that $R p s 27 l$ inactivation causes increased binding of Mdm2-Nbs1 and decreased binding of Nbs1-Atm, leading to reduced Atm activation and DNA damage response upon radiation exposure (Fig. 6b). Our results are in consistence with recent reports that MDM2 may negatively regulate genomic stability independent of p53 by directly binding to Nbs1 of the MRN complex to impair MRN complexmediated ATM activation and subsequent DNA damage response in other model systems ${ }^{21-23,35}$. We also found that Mdm2 indeed plays a causal role in the process, since simultaneous deletion of one $M d m 2$ allele to reduce Mdm2 levels rescued defective Atm activation (Fig. 6c). Given that RPS27L binding region on MDM2 (amino acids 151-293) overlaps with MDM2-binding region (amino acids $198-314$ ) to $\mathrm{NBS1}^{21,36}$, it is very likely that Rps27l depletion vacates the Nbs1-binding region on $\mathrm{Mdm} 2$ to facilitate Mdm2-Nbs1 binding. It would be intriguing to investigate whether other MDM2-binding ribosomal proteins (particularly those reported to maintain genomic stability) with overlapping NBS1-binding region, affect DNA damage response by regulating MDM2-NBS1 binding. Finally, it is worth noting that when $M d m 2$ dose is reduced by one $M d m 2$ allele deletion in Rps $277^{-/-}$; Trp $53^{+/-}$MEFs, we observed a recovery of Mdm4 levels, as well as increased p53 levels (Fig. 6c). The further increase of p53 appears to be sufficient to cause the death, since the life span is significantly shortened in Rps $277^{-/-}$; Trp $53^{+/-} ; M d m 2^{+/-}$mice (Figure S3). Thus, the components of the Rps27l-Mdm2-Mdm4 axis coordinately cross-talk with each other to precisely regulate p53 levels.

In summary, we made here a novel in vivo observation that inactivation of Rps27l in p53 heterozygous background significantly sensitizes mouse to radiation by induction of massive apoptosis in multiple radiation- sensitive organs. Two underlying mechanisms were involved. First, ribosomal stress triggered by Rps $27 l$ inactivation stabilizes $\mathrm{Mdm} 2$ levels, leading to the imbalanced Mdm2 vs. Mdm4 levels and reduced E3 ligase activity toward $\mathrm{p} 53^{19}$. As a result, $\mathrm{p} 53$ levels are increased to induce apoptosis, particularly upon radiation exposure. Second, the increased Mdm2 promotes its Nbs1 binding and consequently reduces Nbs1-Atm binding to inhibit Atm activation, leading to reduced DNA damage response

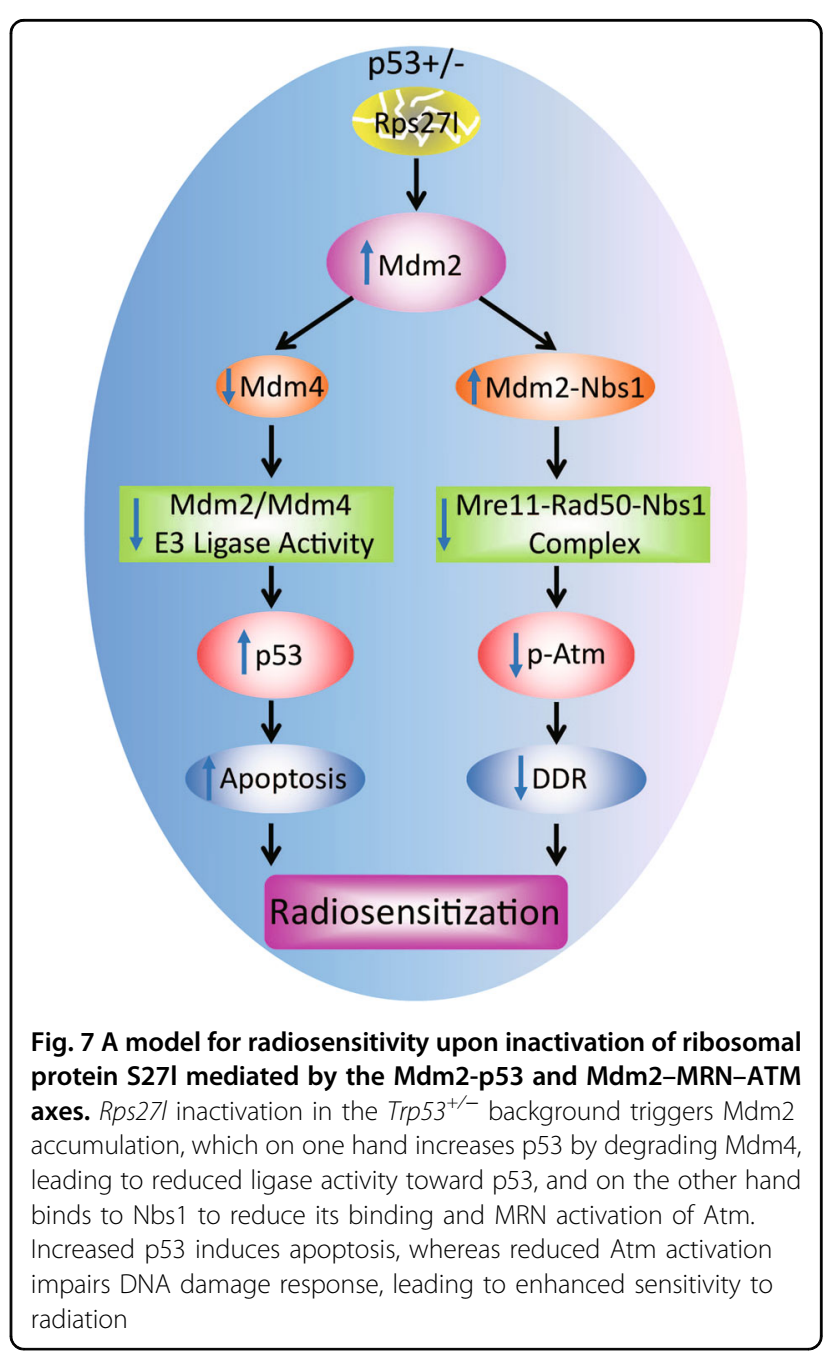


(Fig. 7). Although both mechanisms contribute to increased radiosensitivity, the fact that Rps $27 l$ depletion causes an increased p53 stabilization and activity (Fig. 3f) and a reduced Atm activation (Fig. 4), which would reduce p53 activation, suggests that the $\mathrm{Mdm} 2-\mathrm{Mdm} 4$ axis plays a more important role than the Mdm2-Nbs1-Atm axis in controlling p53 levels and activity under $R p s 27 l^{-1-}$; Trp $53^{+/-}$background. Collectively, our study reveals a physiological relevant condition under which Rps27l regulates the Mdm2-p53 and Mdm2-MRN-ATM axes to maintain DNA damage response and to confer radioprotection. Our study also provides a rationale for enhancing the efficacy of radiation therapy by inactivating RPS27L in human cancers with $\mathrm{TP}^{\mathrm{N}} \mathrm{3}^{+/-}$status.

\section{Materials and methods Mouse studies}

The Rps27l gene-trapped mice and Trp53-deficient mice were obtained and genotyped as previously described $^{19}$. All procedures were approved by the University of Michigan Committee on Use and Care of Animals. Animal care was provided in accordance with the principles and procedures outlined in the National Research Council Guide for the Care and Use of Laboratory Animals.

\section{Radiation exposure and clonogenic assay}

Mice aged at 8-10 weeks were exposed to different doses of radiation (Philips RT250, Kimtron Medical), followed by survival monitor or being sacrificed at indicated time points. Spontaneously immortalized MEFs were seeded in $60 \mathrm{~mm}$ dishes and exposed to different doses of radiation, followed by culture at $37^{\circ} \mathrm{C}$ for 7 days. Survival curves were fitted using the linear quadratic equation and the mean inactivation dose was calculated $^{37,38}$.

\section{Generation and maintenance of MEFs}

MEF cells were generated from day E13.5 embryos with indicated genotypes as described ${ }^{39}$, and cultured in DMEM with 15\% FBS, $2 \mathrm{mM}$ L-glutamine, $0.1 \mathrm{mM}$ MEM non-essential amino acids at $37^{\circ} \mathrm{C}$ in a $5 \% \mathrm{CO}_{2}$ humidified chamber.

\section{Western blotting and immunoprecipitation}

Cells or tissues were collected, lysed, and subjected to western blotting or immunoprecipitation ${ }^{19}$, using various antibodies as follows: RPS27L polyclonal rabbit antibody was raised and purified as described ${ }^{17}$, p53 (1C12 from Cell Signaling), Mdm2 4B2 and Mdm4 7A8 (gifts from Dr. Jiandong Chen), p21 (556430 from BD Pharmingen), Puma (Cell Signaling), $\gamma$ H2AX (JBW301 from Millipore), pho-ATM (Rockland), ATM (Cell Signaling), pho-Chk1 (Cell Signaling), Chk1 (Santa Cruz), NBS1 (Cell
Signaling), rabbit polyclonal Mdm2 Ab for IP raised and purified as described $^{19}$, and $\beta$-actin (Sigma).

\section{Immunohistochemistry}

Tissues were fixed in 10\% formalin and embedded in paraffin. Sections were cut in $5 \mu \mathrm{m}$-thick and subjected to immunohistochemical staining ${ }^{40}$. Briefly, following dewaxing, rehydration, and epitope retrieval, serial sections were labeled with p53 (CM5p, Leica Microsystems), cleaved caspase-3 (Cell Signialing), or $\gamma \mathrm{H} 2 \mathrm{AX}$ (Millipore), followed by staining with Vectastain $\mathrm{ABC}$ kit (Vector Laboratories). Sections were then developed with DAB (Vector Laboratories) and counterstained with haematoxylin. BrdU staining and TUNEL assay were performed using 5-Bromo-2'-deoxy-uridine labeling and detection kit II (Roche) and In Situ Cell Death Detection Kit (Roche), respectively, as manufacturer's instructions.

\section{Statistical analysis}

Statistical analysis was performed using two-tailed Student's $t$-test. Data were expressed as mean \pm SD. Survival analysis was performed by Kaplan-Meier analysis. Statistical significance was determined as $p<0.05$.

\section{Acknowledgements}

We thank Dr. Guillermina Lozano at the University of Texas M.D. Anderson Cancer Center for providing us Mdm2-deficient mice. We also thank Dr. Jiandong Chen for providing antibodies. This work was supported in part by the National Natural Science Foundation of China (Grants No. 81572708 and 31501129 to X.X., 31470753 and 81672728 to Y.Z., and 81572718 and 81630076 to Y.S.), by National Key R\&D Program of China (2016YFA0501800 to Y.S., Y.Z., and X.X.), and by the $\mathrm{NCl}$ grant (CA156744 to Y.S.).

\section{Author details}

'Institute of Translational Medicine, Zhejiang University School of Medicine, Hangzhou, China. ${ }^{2}$ Division of Radiation and Cancer Biology, Department of Radiation Oncology, University of Michigan, Ann Arbor, MI, USA. ${ }^{3}$ Key Laboratory of Combined Multi-Organ Transplantation, Ministry of Public Health, First Affiliated Hospital, Zhejiang University School of Medicine, Hangzhou, China. ${ }^{4}$ Collaborative Innovation Center for Diagnosis and

Treatment of Infectious Diseases, Zhejiang University, Hangzhou, China

\section{Conflict of interest}

The authors declare that they have no conflict of interest.

\section{Publisher's note}

Springer Nature remains neutral with regard to jurisdictional claims in published maps and institutional affiliations.

Supplementary information The online version of this article (https://doi. org/10.1038/s41419-017-0192-3) contains supplementary material.

Received: 29 October 2017 Revised: 27 November 2017 Accepted: 28 November 2017

Published online: 02 February 2018

\footnotetext{
References

1. Ciccia, A. \& Elledge, S. J. The DNA damage response: making it safe to play with knives. Mol. Cell 40, 179-204 (2010).
} 
2. Ghosal, G. \& Chen, J. DNA damage tolerance: a double-edged sword guarding the genome. Transl. Cancer Res. 2, 107-129 (2013).

3. Maréchal, A. \& Zou, L. DNA damage sensing by the ATM and ATR kinases. Cold Spring Harb. Perspect. Biol. 5, a012716 (2013).

4. Uziel, T. et al. Requirement of the MRN complex for ATM activation by DNA damage. EMBO J. 22, 5612-5621 (2003).

5. Lee, J. H. \& Paull, T. T. ATM activation by DNA double-strand breaks through the Mre11-Rad50-Nbs1 complex. Science 308, 551-554 (2005).

6. You, Z., Chahwan, C., Bailis, J., Hunter, T. \& Russell, P. ATM activation and its recruitment to damaged DNA require binding to the $C$ terminus of Nbs1. Mol. Cell Biol. 25, 5363-5379 (2005).

7. Guleria, A. \& Chandna, S. ATM kinase: Much more than a DNA damage responsive protein. DNA Repair 39, 1-20 (2016).

8. Karnitz, L. M. \& Zou, L. Molecular pathways: targeting ATR in cancer therapy. Clin. Cancer Res. 21, 4780-4785 (2015).

9. Smith, J., Tho, L. M., Xu, N. \& Gillespie, D. A. The ATM-Chk2 and ATR-Chk1 pathways in DNA damage signaling and cancer. Adv. Cancer Res. 108, 73-112 (2010).

10. de la Cruz, J., Karbstein, K. \& Woolford, J. L. Jr. Functions of ribosomal proteins in assembly of eukaryotic ribosomes in vivo. Annu. Rev. Biochem. 84, 93-129 (2015).

11. Boulon, S., Westman, B. J., Hutten, S., Boisvert, F. M. \& Lamond, A. I. The nucleolus under stress. Mol. Cell 40, 216-227 (2010).

12. Zhang, Y. \& Lu, H. Signaling top53: ribosomal proteins find their way. Cancer Cell 16, 369-377 (2009).

13. Bursac, S., Brdovcak, M. C., Donati, G. \& Volarevic, S. Activation of the tumor suppressor p53 upon impairment of ribosome biogenesis. Biochim. Biophys. Acta 1842, 817-830 (2014)

14. Zhou, X., Liao, J. M., Liao, W. J. \& Lu, H. Scission of the p53-MDM2 loop by ribosomal proteins. Genes Cancer 3, 298-310 (2012).

15. $\mathrm{Xu}, \mathrm{X} ., \mathrm{Xiong}, \mathrm{X} . \&$ Sun, $\mathrm{Y}$. The role of ribosomal proteins in the regulation of cell proliferation, tumorigenesis, and genomic integrity. Sci. China Life Sci. 59, 656-672 (2016).

16. Vogelstein, B., Lane, D. \& Levine, A. J. Surfing the p53 network. Nature $\mathbf{4 0 8}$ 307-310 (2000)

17. He, H. \& Sun, Y. Ribosomal protein S27L is a direct p53 target that regulates apoptosis. Oncogene 26, 2707-2716 (2007).

18. Li, J. et al. Ribosomal protein S27-like, a p53-inducible modulato of cell fate in response to genotoxic stress. Cancer Res. 67, 11317-11326 (2007).

19. Xiong, $X$. et al. Ribosomal protein S27-like is a physiological regulator of p53 that suppresses genomic instability and tumorigenesis. Elife 3, e02236 (2014).

20. Gudkov, A. V. \& Komarova, E. A. The role of p53 in determining sensitivity to radiotherapy. Nat. Rev. Cancer 3, 117-129 (2003).

21. Alt, J. R. et al. Mdm2 binds to Nbs1 at sites of DNA damage and regulates double strand break repair. J. Biol. Chem. 280, 18771-18781 (2005).

22. Bouska, A., Lushnikova, T., Plaza, S. \& Eischen, C. M. Mdm2 promotes genetic instability and transformation independent of p53. Mol. Cell. Biol. 28 4862-4874 (2008).
23. Bouska, A. \& Eischen, C. M. Mdm2 affects genome stability independent of p53. Cancer Res. 69, 1697-1701 (2009).

24. Llanos, S. \& Serrano, M. Depletion of ribosomal protein L37 occurs in response to DNA damage and activates p53 through the L11/MDM2 pathway. Cell Cycle 9, 4005-4012 (2010).

25. Zhu, Y. et al. Ribosomal protein S7 is both a regulator and substrate of MDM2. Mol. Cell 35, 316-326 (2009).

26. Ofir-Rosenfeld, Y., Boggs, K., Michael, D., Kastan, M. B. \& Oren, M. Mdm2 regulates p53 mRNA translation through inhibitory interactions with ribosomal protein L26. Mol. Cell 32, 180-189 (2008).

27. Cui, D. et al. The ribosomal protein $\mathrm{S} 26$ regulates p53 activity in response to DNA damage. Oncogene 33, 2225-2235 (2014).

28. Kim, T. H., Leslie, P. \& Zhang, Y. Ribosomal proteins as unrevealed caretakers for cellular stress and genomic instability. Oncotarget 5, 860-871 (2014).

29. Fang, S., Jensen, J. P., Ludwig, R. L., Vousden, K. H. \& Weissman, A. M. Mdm2 Is a RING finger-dependent ubiquitin protein ligase for itself and p53. J. Biol. Chem. 275, 8945-8951 (2000).

30. Haupt, Y., Maya, R., Kazaz, A. \& Oren, M. Mdm2 promotes the rapid degradation of p53. Nature 387, 296-299 (1997).

31. Kubbutat, M. H. G., Jones, S. N. \& Vousden, K. H. Regulation of p53 stability by Mdm2. Nature 387, 299-303 (1997).

32. Huang, L. et al. The p53 inhibitors MDM2/MDMX complex is required for control of p53 activity in vivo. Proc. Natl Acad. Sci. USA 108, 12001-12006 (2011).

33. Pant, V., Xiong, S., Iwakuma, T., Quintás-Cardama, A. \& Lozano, G. Heterodimerization of Mdm2 and Mdm4 is critical for regulating p53 activity during embryogenesis but dispensable for p53 and Mdm2 stability. Proc. Natl Acad. Sci. USA 108, 11995-12000 (2011).

34. Wang, $X$. Wang, J. \& Jiang, X. MdmX protein is essential for Mdm2 Protein-mediated p53 polyubiquitination. J. Biol. Chem. 286, 23725-23734 (2011).

35. Wang, P. et al. Elevated Mdm2 expression induces chromosomal instability and confers a survival and growth advantage to B cells. Oncogene 27, 1590-1598 (2007)

36. Xiong, X., Zhao, Y., He, H. \& Sun, Y. Ribosomal protein S27-like and S27 interplay with p53-MDM2 axis s a target, a substrate, and a regulator. Oncogene 30, 1798-1811 (2011).

37. Yang, J. et al. Radiosensitization of head and neck squamous cell carcinoma by a SMAC-mimetic compound, SM-164, requires activation of caspases. Mol. Cancer Ther. 10, 658-669 (2011).

38. Zhang, Q. et al. FBXW7 facilitates nonhomologous end-joining via K63-linked polyubiquitylation of XRCC4. Mol. Cell 61, 419-433 (2016).

39. Tan, M., Davis, S. W., Saunders, T. L., Zhu, Y. \& Sun, Y. RBX1/ROC1 disruption results in early embryonic lethality due to proliferation failure, partially rescued by simultaneous loss of p27. Proc. Natl Acad. Sci. USA 106, 6203-6208 (2009)

40. Tan, M., Xu, J., Siddiqui, J., Feng, F. \& Sun, Y. Depletion of SAG/RBX2 E3 ubiquitin ligase suppresses prostate tumorigenesis via inactivation of the PI3K AKT/mTOR axis. Mol. Cancer 15, 81 (2016). 